\title{
SOME SPECTRAL PROPERTIES OF THE NON-SELF-ADJOINT FRIEDRICHS MODEL OPERATOR
}

\author{
By Alexander V. Kiselev* \\ School of Mathematical Sciences, Dublin Institute of Technology \\ and
}

Department of Mathematical Physics, St. Petersburg State University, Ulianovskaya, 1, Peterhoff, St. Petersburg, 198504 Russia

[Received 19 December 2003. Read 31 January 2005. Published 12 July 2005.]

To Katusha with love

\section{Abstract}

A non-self-adjoint, rank-one Friedrichs model operator in $L_{2}(\mathbb{R})$ is considered in the case where the determinant of perturbation is an outer function in the half-planes $\mathbb{C}_{+}$. Its spectral structure is investigated. The impact of the linear resolvent growth condition on its spectral properties (including the similarity problem) is studied.

\section{Introduction}

We consider a non-self-adjoint Friedrichs model operator in the Hilbert space $L_{2}(\mathbb{R})$, i.e., a rank-one perturbation $L$ of the multiplication operator, defined by

$$
(L u)(x)=x u(x)+\langle u, \varphi\rangle \psi(x), \quad u, \varphi, \psi \in L_{2}(\mathbb{R}) .
$$

The spectral properties of the operator (1.1) have been studied (see $[3 ; 4 ; 5 ; 9 ; 10$; 15], for example), and a number of results have been obtained, especially concerning sufficient conditions for its similarity to a self-adjoint operator - an operator $L$ is said to be similar to a self-adjoint one if there exist a self-adjoint operator $A$ and a bounded, boundedly invertible operator $X$ such that $\left.L=X^{-1} A X\right)$.

Such sufficient conditions were formulated in their simplest terms in $[3 ; 4]$ in two particular cases, $\bar{\varphi} \psi=0$ a.e. and $\bar{\varphi} \psi \geq 0$ a.e., respectively. These results were obtained using the resolvent test $[9 ; 14]$ : an operator $L$, acting in the Hilbert space $H$, is similar to a self-adjoint operator if and only if there exists a finite positive constant $C$ such that

$$
\begin{gathered}
\sup _{\varepsilon>0} \varepsilon \int_{-\infty}^{\infty}\left\|(L-k-i \varepsilon)^{-1} u\right\|^{2} d k \leq C\|u\|^{2} \\
\sup _{\varepsilon>0} \varepsilon \int_{-\infty}^{\infty}\left\|\left(L^{*}-k-i \varepsilon\right)^{-1} u\right\|^{2} d k \leq C\|u\|^{2}
\end{gathered}
$$

*E-mail: alexander.kiselev@dit.ie, akiselev@mph.phys.spbu.ru 
for all $u \in H$.

In the present paper, we prove some new results concerning the spectral analysis of the operator $L$ and establish the impact of some well-known concise tests, most notably, the Sahnovich condition and the linear resolvent growth condition (LRG)

$$
\left\|(L-\lambda)^{-1}\right\| \leq \frac{C}{|\operatorname{Im} \lambda|}, \quad \operatorname{Im} \lambda \neq 0
$$

on the similarity problem and the structure of the operator's spectrum. The implications of the condition (1.3) on the similarity problem have been treated very recently by Nikolski and Treil (see [11]), and some new, exciting results have been obtained in this area.

The paper is organised as follows:

In the second section, we concentrate our attention on the case when $\bar{\varphi} \psi=0$ a.e. We revisit the results obtained in [4], providing an explicit description of the sets of 'smooth vectors' [7] for both $L$ and its adjoint, $L^{*}$, under the assumption of similarity of $L$ to a self-adjoint operator. We also prove that in this case the spectra of the operator $L$ and $L^{*}$ are absolutely continuous, and we study the implications of this spectral structure for the similarity problem and the estimates (1.2).

In the third section, we focus on the case where $\overline{\varphi(x)} \psi(x) \geq 0$ a.e. In this case, the spectral structure of the operator $L$ becomes much more complicated. In particular, a singular continuous subspace (possibly intersecting with the absolutely continuous one, see [15]) can be present, so our aim is to study the geometry of the singular and absolutely continuous subspaces. We provide a characterisation of the singular spectral subspace $N_{i}^{0}$ of the operator $L$ in terms of a singular integral equation in $L_{2}(\mathbb{R})$. We then derive a straightforward description for the sets of 'smooth' vectors associated with the operators $L$ and $L^{*}$. Finally, we prove a kind of spectral alternative that establishes the impact of the linear resolvent growth condition (1.3) on the spectral structure of the operator $L$ in the presence of an eigenvector corresponding to a real eigenvalue; in particular, the spectral alternative prevents eigenvectors of the operator $L$ from belonging to the absolutely continuous spectral subspace under the LRG condition.

In the fourth section, we describe implications of the LRG condition (1.3) (and of its weaker, 'limit' version) on the spectral structure of the operator $L$ under the assumption that the LRG condition possesses a countable number of real eigenvalues. In particular, we prove that the LRG condition guarantees that, in this case, normalised eigenfunctions form a Riesz basis in the singular subspace $N_{i}^{0}$ and that the angle between the singular and absolutely continuous subspaces of the operator $L$ is positive.

\section{Spectral properties in the case of disjoint supports}

Throughout this section we will assume that the functions $\varphi$ and $\psi$ determining the perturbation have disjoint supports, i.e., $\bar{\varphi} \psi=0$ almost everywhere on the real axis.

In [4] the following result was obtained. Let $H=L_{2}(\mathbb{R})$. Let $A$ denote the self-adjoint multiplication operator in $H$ on its natural domain, $(A u)(x):=x u(x)$. 
Consider the family of bounded integral operators $T_{\varepsilon}$ in $H$, defined by their respective kernels $T_{\varepsilon}(x, y):=\frac{\psi(x) \bar{\varphi}(y)}{x-y-i \varepsilon}$ and the (in general cases, unbounded) singular integral operator $T$ in $H$, defined by its kernel $T(x, y):=\frac{\psi(x) \bar{\varphi}(y)}{x-y}$. Clearly, the operator $T$ is bounded in $H$ if and only if the norms of the operators $T_{\varepsilon}$ are uniformly bounded and, in this case, $T_{\varepsilon} \stackrel{w}{\longrightarrow} T$ by the Banach-Steinhaus theorem.

Then the operator (1.1) with $\bar{\varphi} \psi=0$ a.e. is similar to a self-adjoint operator if and only if the operator $T$ is bounded. Moreover, one has the following formula for the non-orthogonal spectral measure $P$ of $L$ :

$$
P(\delta)=E(\delta)-(T E(\delta)-E(\delta) T),
$$

where $\delta$ is an arbitrary interval of the real axis and $E$ is the spectral measure associated with the operator $A$. The operator $L$ itself then possesses the following representation:

$$
L=A-T A+A T .
$$

We now extend this result in the following way:

Proposition 2.1. Suppose that $\bar{\varphi} \psi=0$ a.e., then:

(i) if the operator $T$ is bounded in $L_{2}(\mathbb{R})$, then the operator $L$ is similar to the operator $A$, i.e., $L=X^{-1} A X$, where $X=T+\mathbf{1}$;

(ii) if the operator $T$ is unbounded, there exists a sequence of operators $L_{n}$ of the form (1.1), such that each of the operators $L_{n}$ is similar to $A ; L-L_{n}$ is a bounded operator in $L_{2}(\mathbb{R})$; and $\left\|L-L_{n}\right\| \rightarrow 0$ as $n \rightarrow \infty$.

Proof. (i) Note that, clearly, $T^{2}=0$ since $\bar{\varphi} \psi=0$ a.e. Then $(T+\mathbf{1})^{-1}=\mathbf{1}-T$ and

$$
\begin{aligned}
(\mathbf{1}-T) A(T+\mathbf{1}) u(x)=x \psi(x) \mathcal{X} \frac{\bar{\varphi}(y) u(y)}{x-y} d y+ & x u(x)-\psi(x) \bigvee \frac{\bar{\varphi}(y) y u(y)}{x-y} d y \\
& =x u(x)+\psi(x) \int u(y) \bar{\varphi}(y) d y .
\end{aligned}
$$

The symbol $\int$ (or, alternatively, V.P. $\int$ ) is used throughout the present paper to denote the Cauchy mean value integral over the real axis.

(ii) Let $L$ be an operator of the form (1.1), and let $\varphi, \psi \in L_{\infty}(\mathbb{R})$. Then the operator $L$ is similar to the self-adjoint operator $A$, since by the Holder inequality and M. Riesz theorem [6]

$$
\begin{aligned}
|\langle T u, v\rangle| & =\left|\int \psi(x) \bar{v}(x)\left(\int \frac{\bar{\varphi}(y) u(y)}{x-y} d y\right) d x\right| \\
\leq & \left(\int|\psi(x) v(x)|^{2} d x\right)^{1 / 2}\left(\int|\varphi(x) u(x)|^{2} d x\right)^{1 / 2} \leq\|\psi\|_{L_{\infty}}\|\varphi\|_{L_{\infty}}\|u\|\|v\|
\end{aligned}
$$

for all $u, v \in L_{2}(\mathbb{R})$, and therefore the operator $T$ is bounded in $L_{2}(\mathbb{R})$.

We now choose two sequences of bounded functions $\left\{\varphi_{n}\right\}$ and $\left\{\psi_{n}\right\}$ such that 
$\varphi_{n} \rightarrow \varphi$ and $\psi_{n} \rightarrow \psi$ in $L_{2}(\mathbb{R})$ as $n \rightarrow \infty$, and a corresponding sequence of the operators $L_{n}$, defined as follows:

$$
\left(L_{n} u\right)(x):=x u(x)+\left\langle u, \varphi_{n}\right\rangle \psi_{n}(x) .
$$

The operators $L_{n}$ satisfy the required property, since

$$
L-L_{n}=\left\langle\cdot, \varphi-\varphi_{n}\right\rangle \psi-\left\langle\cdot, \varphi_{n}\right\rangle\left(\psi-\psi_{n}\right) .
$$

We proceed with establishing the spectral properties of the operator $L$. Following $[8 ; 15]$ (see also references therein), we define the spectral subspaces of the operator under investigation as follows:

Let $L=\operatorname{Re} L+i \operatorname{Im} L$, where $\operatorname{Re} L=\left(L+L^{*}\right) / 2$ and $\operatorname{Im} L=\left(L-L^{*}\right) / 2 i$ are self-adjoint operators. (For the sake of simplicity, we assume without any loss of generality, that $L$ is completely non-self-adjoint, i.e., has no reducing self-adjoint parts). Consider the polar decomposition of the operator $V \equiv \operatorname{Im} L$ of the following form: $V=J \frac{\alpha^{2}}{2}$, where $J \equiv \operatorname{sign} V$ and $\alpha \equiv \sqrt{2|V|}$. Furthermore, let $E=\operatorname{clos}(\operatorname{Range}(\alpha))$ be an auxiliary Hilbert space and let the projectors in it be chosen as follows: $\mathcal{X}_{ \pm} \equiv(I \pm J) / 2$.

Consider the linear sets $\tilde{N}_{ \pm}$in $H$, defined by the following formula:

$$
\tilde{N}_{ \pm}=\left\{u \in H: \mathcal{X}_{ \pm} \alpha(L-\lambda)^{-1} u \in H_{2}^{ \pm}(E)\right\},
$$

where $H_{2}^{ \pm}(E)$ denote the Hardy classes of $E$-valued analytic functions in the upper (respectively, lower) half-plane of the complex plane [13]. Let $\tilde{N}_{e} \equiv \tilde{N}_{-} \cap \tilde{N}_{+}$. The linear set $\tilde{N}_{e}$ is called [7] the set of smooth vectors associated with the operator $L$.

Then the absolutely continuous subspace $N_{e}$ of the operator $L$ is defined as the closure in $H$ of $\tilde{N}_{e}: N_{e} \equiv \operatorname{clos}\left(\tilde{N}_{e}\right)$. The singular subspace $N_{i}$ of $L$ is defined as follows: $N_{i} \equiv H \ominus N_{e}^{*}$, where $N_{e}^{*}$ is the absolutely continuous subspace of the adjoint operator $L^{*}$.

In view of what follows, we also define the singular subspace $N_{i}^{0} \equiv H \ominus\left(N_{+}^{*} \wedge\right.$ $N_{-}^{*}$ ), where

$$
N_{ \pm}^{*}=\operatorname{clos}\left\{u \in H: \mathcal{X}_{\mp} \alpha\left(L^{*}-\lambda\right)^{-1} u \in H_{2}^{ \pm}(E)\right\}
$$

are the subspaces associated with $L^{*}$, which are analogous to $N_{ \pm} \equiv \operatorname{clos}\left(\tilde{N}_{ \pm}\right)$for the operator $L$. By [15] $N_{i}^{0} \subset N_{i}$ and $N_{i}^{0}$ consists of, at least, all eigenvectors and root vectors of $L$ corresponding to real values of the spectral parameter $\lambda$.

In the case when $\overline{\varphi(x)} \psi(x) \geq 0$ a.e., it is clear that $N_{i}^{0}=N_{i}[15]$.

Now we are ready to prove the following proposition:

Proposition 2.2. Suppose that $\bar{\varphi} \psi=0$ a.e. and the operator $T$ is bounded in $L_{2}(\mathbb{R})$. Then:

(i) The absolutely continuous subspace $N_{e}$ of $L$ is equal to $L_{2}(\mathbb{R})$, and the cor- 
responding dense linear set $\tilde{N}_{e}$ can be chosen as follows:

$$
\tilde{N}_{e}=(T+\mathbf{1}) L_{\infty}(\mathbb{R}) .
$$

(ii) The absolutely continuous subspace $N_{e}^{*}$ of $L^{*}$ is equal to $L_{2}(\mathbb{R})$, and the corresponding dense linear set $\tilde{N}_{e}^{*}$ can be chosen as follows:

$$
\tilde{N}_{e}^{*}=\left(\mathbf{1}-T^{*}\right) L_{\infty}(\mathbb{R})
$$

Proof. We are going to prove the assertion (i), since (ii) can be proved in an absolutely analogous way.

Since $\bar{\varphi} \psi=0$ a.e., and therefore $\langle\varphi, \psi\rangle=0$, the operator $\alpha$ is a diagonal operator in $E$ :

$$
\begin{gathered}
\alpha=\left(\frac{\|\psi\|}{\|\varphi\|}\right)^{1 / 2} \frac{1}{\|\varphi\|}\langle\cdot, \varphi\rangle \varphi+\left(\frac{\|\varphi\|}{\|\psi\|}\right)^{1 / 2} \frac{1}{\|\psi\|}\langle\cdot, \psi\rangle \psi \\
J=\frac{1}{i\|\varphi\|\|\psi\|}(\langle\cdot, \varphi\rangle \psi-\langle\cdot, \psi\rangle \varphi) .
\end{gathered}
$$

Furthermore, the resolvent $(L-\lambda)^{-1}$ satisfies the following identity:

$$
\left((L-\lambda)^{-1} u\right)(x)=\frac{u(x)}{x-\lambda}-\left\langle\frac{u(t)}{t-\lambda}, \varphi(t)\right\rangle \frac{\psi(x)}{x-\lambda} .
$$

A straightforward calculation now shows that all we need to prove is that

$$
\left\langle\frac{u(x)}{x-\lambda}, \varphi(x)\right\rangle \text { and }\left\langle\frac{u(x)}{x-\lambda}, \psi(x)\right\rangle-\left\langle\frac{u(t)}{t-\lambda}, \varphi(t)\right\rangle\left\langle\frac{\psi(x)}{x-\lambda}, \psi(x)\right\rangle
$$

both belong to $H_{2}^{ \pm}$when $u \in(T+\mathbf{1}) L_{\infty}(\mathbb{R})$.

Since the functions $u$ belonging to this linear set are clearly bounded on the support of the function $\varphi$ due to the definition of the operator $T$ so that $u \varphi \in L_{2}$, the first function in $(2.3)$ belongs to $H_{2}^{ \pm}$[6].

The second function in (2.3) can be rewritten in the following way:

$$
\begin{aligned}
\left\langle\frac{u(x)}{x-\lambda}, \psi(x)\right\rangle-\left\langle\frac{u(t)}{t-\lambda}, \varphi(t)\right\rangle\left\langle\frac{\psi(x)}{x-\lambda}, \psi(x)\right\rangle \\
=\int \frac{u(t) \bar{\varphi}(t)}{t-\lambda}\left(\int \frac{|\psi|^{2}}{x-t} d x\right) d t-\int \frac{\bar{\psi}(x)(T-\mathbf{1}) u(x)}{x-\lambda} d x \\
=\int \frac{u(t) \bar{\varphi}(t)}{t-\lambda}\left(\int \frac{|\psi|^{2}}{x-t} d x\right) d t+\int \frac{\bar{\psi}(x) f(x)}{x-\lambda} d x
\end{aligned}
$$

where $f=(T-\mathbf{1}) u \in L_{\infty}(\mathbb{R})$. Since $\bar{\varphi}(t) \hat{\gamma}|\psi(x)|^{2} /(x-t) d x \equiv-\overline{T^{*} \psi} \in L_{2}$ and $u \in L_{\infty}(\operatorname{supp} \varphi)$, the first integral in the last expression belongs to $H_{2}^{ \pm}$, and so does the second, since $\bar{\psi} f \in L_{2}(\mathbb{R})$, by the Riesz theorem [6].

We now prove that the linear set $(T+\mathbf{1}) \varphi$ is dense in $L_{2}(\mathbb{R})$. Consider an 
arbitrary element $u \in L_{2}$. Let a sequence of bounded functions $\left\{f_{n}\right\}$ be chosen so that $f_{n} \rightarrow(1-T) u \in L_{2}(\mathbb{R})$ as $n \rightarrow \infty$. Then, using the boundedness of the operator $T$ once again, $(\mathbf{1}+T) f_{n} \rightarrow(\mathbf{1}+T)(\mathbf{1}-T) u \equiv u$ as $n \rightarrow \infty$, which completes the proof.

Remark 2.1. Given that the operator $L$ is similar to a self-adjoint operator with purely absolutely continuous spectrum, the identity $N_{e}=N_{e}^{*}=H$ can in fact be verified in the general case along the lines of the proof of [15, proposition 3]. Our proof above takes advantage of the simplicity of the Friedrichs model resolvent $(L-\lambda)^{-1}$ and thus allows us to describe the set of smooth vectors of the operator and its adjoint explicitly.

In the general case of $\bar{\varphi} \psi \neq 0$, Proposition 2.2 no longer holds (see Section 3 ).

In fact, in the case when $\bar{\varphi} \psi=0$ a.e. the operators $L$ and $L^{*}$ always have purely absolutely continuous spectra.

Proposition 2.3. Let $\bar{\varphi} \psi=0$ a.e. Then $N_{e}=N_{e}^{*}=H$.

Proof. By [15], a vector $u$ belongs to the singular spectral subspace $N_{i}$ if and only if

$$
\left\langle\left[(L-\lambda)^{-1}-(L-\bar{\lambda})^{-1}\right] u, v\right\rangle \rightarrow 0 \text { as } \varepsilon \rightarrow 0 \text { for a.a. } k(\lambda=k+i \epsilon)
$$

for all $v \in H$.

Using the expression (2.2), we obtain

$$
\begin{aligned}
&\left\langle\left[(L-\lambda)^{-1}-(L-\bar{\lambda})^{-1}\right] u, v\right\rangle=2 i \pi \mathcal{P}[u \bar{v}](k+i \varepsilon)- \\
&\left\langle\frac{u(t)}{t-\lambda}, \varphi(t)\right\rangle\left\langle\frac{\psi(x)}{x-\lambda}, v(x)\right\rangle+\left\langle\frac{u(t)}{t-\bar{\lambda}}, \varphi(t)\right\rangle\left\langle\frac{\psi(x)}{x-\bar{\lambda}}, v(x)\right\rangle,
\end{aligned}
$$

where $\mathcal{P}[f]$ denotes the Poisson transform (i.e., the integral transformation with the Poisson kernel, [6]) of the function $f$.

Then

$$
\left\langle\left[(L-\lambda)^{-1}-(L-\bar{\lambda})^{-1}\right] u, v\right\rangle \rightarrow 2 i \pi(u(k) \bar{v}(k)-\psi(k) \bar{v}(k) \widetilde{u \bar{\varphi}}(k)-u(k) \bar{\varphi}(k) \widetilde{\psi} \bar{v}(k))
$$

for a.a. $k$ (see [6]), where we used the notation

$$
\tilde{f}(t) \equiv \lim _{\varepsilon \rightarrow 0} \int \frac{\tau-k}{(\tau-k)^{2}+\varepsilon^{2}} f(\tau) d \tau=\chi \frac{f(\tau)}{\tau-k} d \tau
$$

for any function $f$ on the real axis such that the right-hand side makes sense.

Let $u \in N_{i}$. Then $u$ satisfies the following equation almost everywhere on the real axis:

$$
u(k) \bar{v}(k)-\psi(k) \bar{v}(k) \widetilde{u \bar{\varphi}}(k)-u(k) \bar{\varphi}(k) \widetilde{\psi} \bar{v}(k)=0 .
$$


Multiplying this equation by $\bar{\varphi}(k)$ and choosing $v$ such that $\psi(x) \bar{v}(x)=0$ a.e., we conclude that $u \equiv 0$ on support of the function $\varphi$.

Multiplying $(2.5)$ by $\psi(k)$, we then conclude that $u \equiv 0$ on the support of the function $\psi$ as well, which leaves us with the identity $u(k) \bar{v}(k)=0$ a.e. for all functions $v \in L_{2}(\mathbb{R})$, and therefore, necessarily, $u \equiv 0$ and $N_{i}=\{0\}$.

Since, quite analogously, $N_{i}^{*}=\{0\}$, this completes the proof.

Consider now the implications of the spectral structure of $L$ provided by Proposition 2.3 on the integral estimates (1.2).

Proposition 2.4. Let $\bar{\varphi} \psi=0$ a.e. Then the first estimate in (1.2) is equivalent to the second one (i.e., if the first estimate in (1.2) holds for all $u \in H$, then the second one holds for all $u \in H$, and vice versa).

Proof. Consider the functional model representation for the operator $L$ (see [7]). There exists a Hilbert space $\mathcal{H} \supset H$ such that

$$
(L-\lambda)^{-1} u=P_{H}(k-\lambda)^{-1} \hat{u}
$$

where $u=P_{H} \hat{u}, P_{H}$ denoting the orthogonal projection onto $H$, for all $u \in \tilde{N}_{e}$ (the set of smooth vectors of the operator $L$ ) and for all $\lambda$ such that $\operatorname{Im} \lambda \neq 0$. The same identity holds for the operator $L^{*}$ on all $u \in \tilde{N}_{e}^{*}$. This argument completes the proof, since by Proposition $2.3 N_{e}=N_{e}^{*}=H$, and hence the linear sets $\tilde{N}_{e}$ and $\tilde{N}_{e}^{*}$ are both dense in $H$.

Remark 2.2. Note that the last result is of a general nature. Indeed, the proof given is applicable (see [7] and references therein) to non-self-adjoint operators of the form $L=A+i V$, where $A, V$ are self-adjoint operators in $H$ defined on the domains $D(A)$ and $D(V)$, respectively; the perturbation $V$ is $(A)$-bounded, with the relative bound less than 1, i.e. for $D(A) \subset D(V)$ and for some $a$ and $b(a<1)$ the condition

$$
\|V u\| \leq a\|A u\|+b\|u\|, \quad u \in D(A)
$$

is satisfied. In this case the operator $L$ is well-defined on the domain $D(L)=D(A)$.

Our next goal is to study the implications of some well-known concise tests on the condition of similarity of $L$ to a self-adjoint operator.

First, we recall that the characteristic function $\Theta(\lambda)$ of the operator $L$ is a meromorphic operator function acting in the auxiliary Hilbert space $E$ and defined by the following identity:

$$
\Theta(\lambda)=\mathbf{1}+i J \alpha\left(L^{*}-\lambda\right)^{-1} \alpha, \quad \operatorname{Im} \lambda \neq 0,
$$

and the characteristic function $S(\lambda)$ of the dissipative operator $L^{\|} \equiv R e L+i \alpha^{2} / 2$ is an analytic operator-function in $E$, contractive in the upper half-plane and defined by

$$
S(\lambda)=\mathbf{1}+i \alpha\left(L^{-\|}-\lambda\right)^{-1} \alpha, \quad \operatorname{Im} \lambda>0,
$$


where $L^{-\|} \equiv\left(L^{\|}\right)^{*}$.

Recall that in [8] the following theorem is proved: Let

$$
\sup _{\operatorname{Im} \lambda \neq 0}\left\|\mathcal{X}_{+} \Theta(\lambda) \mathcal{X}_{-}\right\|<1
$$

or

$$
\sup _{\operatorname{Im}} \max \left\{\left\|\mathcal{X}_{+} S(\lambda) \mathcal{X}_{-}\right\|,\left\|\mathcal{X}_{-} S(\lambda) \mathcal{X}_{+}\right\|\right\}<1 .
$$

Then the LRG condition (1.3) is necessary and sufficient for the operator $L$ to be similar to a self-adjoint operator. (Note that (2.6) is sufficient for $(2.7$, see [8]). The reverse implication is false in general; moreover, one can easily present (very much along the lines of the proof of the next proposition) a corresponding counterexample in the case of the Friedrichs model operator as well.)

First of all, we are going to provide an example showing that neither of the conditions (2.7) and (2.6) is tight.

Example 2.5. There exists a pair of functions $\varphi, \psi$, such that

(i) $\bar{\varphi} \psi=0$ a.e. on $\mathbb{R}$;

(ii) the operator $L$ (1.1) is similar to a self-adjoint operator $A$ (and therefore, (1.3) holds);

(iii) the condition (2.7) fails (and therefore, the condition (2.6) fails, too).

Proof. Without any loss of generality, let $\|\varphi\|=\|\psi\|=1$. Based on the identity for the resolvent of the operator $L^{*}$, analogous to $(2.2)$, it is not hard to see that in the orthonormal basis $\{\varphi, \psi\}$ the characteristic function $\Theta(\lambda)$ has the following matrix representation:

$$
\Theta(\lambda)=\left(\begin{array}{cc}
1 & -\left\langle\frac{\psi(t)}{t-\lambda}, \psi(t)\right\rangle \\
\left\langle\frac{\varphi(t)}{t-\lambda}, \varphi(t)\right\rangle & 1-\left\langle\frac{\varphi(t)}{t-\lambda}, \varphi(t)\right\rangle\left\langle\frac{\psi(t)}{t-\lambda}, \psi(t)\right\rangle
\end{array}\right) .
$$

Then, using the following identities [8]

$$
\begin{gathered}
\left(\mathcal{X}_{-}+S(\lambda) \mathcal{X}_{+}\right)^{-1}=\mathcal{X}_{-}+\Theta^{*}(\bar{\lambda}) \mathcal{X}_{+}, \\
\left(\mathcal{X}_{+}-S(\lambda) \mathcal{X}_{-}\right)^{-1}=\mathcal{X}_{+}-\Theta(\lambda) \mathcal{X}_{-}
\end{gathered}
$$

one can obtain the matrix representation of the characteristic function $S(\lambda)$ in the 'symmetric' basis (cf. Proposition 3.2 below.) $\left\{\frac{i \varphi+\psi}{\sqrt{2}}, \frac{-i \varphi+\psi}{\sqrt{2}}\right\}$ : 


$$
S(\lambda)=\frac{1}{M(\lambda)} \times
$$

$$
\times\left(\begin{array}{cc}
2 i & \left\langle\frac{\varphi(t)}{t-\lambda}, \varphi(t)\right\rangle-\left\langle\frac{\psi(t)}{t-\lambda}, \psi(t)\right\rangle- \\
& -i\left\langle\frac{\varphi(t)}{t-\lambda}, \varphi(t)\right\rangle\left\langle\frac{\psi(t)}{t-\lambda}, \psi(t)\right\rangle \\
\left\langle\frac{\varphi(t)}{t-\lambda}, \varphi(t)\right\rangle-\left\langle\frac{\psi(t)}{t-\lambda}, \psi(t)\right\rangle+ & \\
+i\left\langle\frac{\varphi(t)}{t-\lambda}, \varphi(t)\right\rangle\left\langle\frac{\psi(t)}{t-\lambda}, \psi(t)\right\rangle & 2 i
\end{array}\right)
$$

where

$$
M(\lambda)=2 i+\left\langle\frac{\varphi(t)}{t-\lambda}, \varphi(t)\right\rangle+\left\langle\frac{\psi(t)}{t-\lambda}, \psi(t)\right\rangle-i\left\langle\frac{\varphi(t)}{t-\lambda}, \varphi(t)\right\rangle\left\langle\frac{\psi(t)}{t-\lambda}, \psi(t)\right\rangle .
$$

These formulae yield:

$$
\begin{gathered}
\left\|\mathcal{X}_{+} \Theta(\lambda) \mathcal{X}_{-}\right\|=\frac{1}{2}\left|r_{\varphi}-r_{\psi}-i r_{\varphi} r_{\psi}\right| \\
\left\|\mathcal{X}_{+} S(\lambda) \mathcal{X}_{-}\right\|=\frac{\left|i r_{\varphi}-i r_{\psi}+r_{\varphi} r_{\psi}\right|}{\left|2-i r_{\varphi}-i r_{\psi}-r_{\varphi} r_{\psi}\right|}
\end{gathered}
$$

where $r_{\varphi}:=\langle\varphi(t) /(t-\lambda), \varphi(t)\rangle, r_{\psi}:=\langle\psi(t) /(t-\lambda), \psi(t)\rangle$ and both functions have non-negative imaginary parts when $\lambda \in \mathbb{C}_{+}$. Clearly in this case the function (2.11) tends to 1 when the following conditions are met:

$$
\left|r_{\varphi}\right| \rightarrow \infty, \quad\left|\frac{r_{\psi}}{r_{\varphi}}\right| \rightarrow 0 \quad \text { as } \lambda \rightarrow \lambda_{0} \in \mathbb{R}
$$

and in this case the condition (2.7) is violated. Now let $\varphi=\mathcal{X}_{[-1,0]}(x), \psi=\mathcal{X}_{[1,2]}(x)$. Then $\left|r_{\varphi}\right|=|\ln (\lambda /(\lambda+1))| \rightarrow \infty$ as $\lambda \rightarrow 0$, whereas $\left|r_{\psi}\right|=\left|\ln \frac{2-\lambda}{1-\lambda}\right|$ is bounded as $\lambda \rightarrow 0$. Therefore, the chosen functions satisfy the conditions (2.12). On the other hand, the operator $L$ with these functions $\varphi$ and $\psi$ is similar to the self-adjoint operator $A$, since the corresponding operator $T$ is bounded in $L_{2}(\mathbb{R})$.

Recall [12], that for dissipative operators $L$, the condition $\|\Theta(\lambda)\| \leq C<\infty$ for all $\lambda$ such that $\operatorname{Im} \lambda \neq 0$ is necessary and sufficient for the similarity of operator $L$ to a self-adjoint one. If, however, the operator $L$ is non-dissipative, this condition is no longer necessary (although still sufficient) ( see [1]). We are able to construct a corresponding counterexample in our case.

Example 2.6. There exists a pair of functions $\varphi$ and $\psi, \bar{\varphi} \psi=0$ a.e. on $\mathbb{R}$, such that the corresponding operator $L(1.1)$ is similar to a self-adjoint operator $A$, yet the condition $\|\Theta(\lambda)\| \leq C<\infty$ is violated. 
Proof. Let $\varphi=\mathcal{X}_{[-1,0]}(x)$ and $\psi=\mathcal{X}_{[0,1]}(x)$. Clearly, the operator $L$ is similar to a self-adjoint one. Suppose that the operator-function $\Theta(\lambda)$ is uniformly bounded when $\operatorname{Im} \lambda \neq 0$. Then the operator-function $\mathcal{X}_{+} \Theta(\lambda) \mathcal{X}_{-}$is also bounded. But the norm of this operator-function is equal to the expression in (2.10) and tends to $\infty$ as $\lambda \rightarrow 0$.

\section{Spectral structure in the case when the determinant of perturbation is a Nevanlinna function}

In this section we consider the case when $\bar{\varphi} \psi \geq 0$ a.e. on the real axis, so that the determinant of perturbation $D(\lambda)$ (see (3.3)) is a Nevanlinna function [6].

First, we will prove the following characterisation of the singular spectral subspace of the non-self-adjoint Friedrichs model operator $L$ defined by (1.1).

Theorem 3.1. Let $\overline{\varphi(x)} \psi(x) \geq 0$ for a.a. $x \in \mathbb{R}$. Then

(i) If $u \in N_{i}^{0}$ and $\operatorname{supp} u \neq \mathbb{R}$, then $u$ is an $L_{2}$-solution to the singular integral equation

$$
u(k) \overline{\varphi(k)}=\frac{\overline{\varphi(k)} \psi(k)}{1+V . P . \int \frac{\overline{\varphi(t)} \psi(t)}{t-k} d t} V . P . \int \frac{u(t) \overline{\varphi(t)}}{t-k} d t .
$$

(ii) If $u \in L_{2}(\mathbb{R})$ is a solution to the equation (3.1), then $u \in N_{i}^{0}$.

Proof. By [15], a vector $u$ belongs to the singular spectral subspace $N_{i}$, which is in our case equal to $N_{i}^{0}$, if and only if the condition (2.4) holds.

In our case, the resolvent is given by the following expression:

$$
\left((L-\lambda)^{-1} u\right)(x)=\frac{u(x)}{x-\lambda}-\frac{1}{D(\lambda)}\left\langle\frac{u(t)}{t-\lambda}, \varphi(t)\right\rangle \frac{\psi(x)}{x-\lambda},
$$

where the determinant of perturbation $D(\lambda)$ is defined by

$$
D(\lambda)=1+\left\langle\frac{\psi(t)}{t-\lambda}, \varphi(t)\right\rangle=1+\int \frac{\overline{\varphi(t)} \psi(t)}{t-\lambda} d t .
$$

Then

$$
\begin{gathered}
\frac{1}{D(\lambda)}\left\langle\frac{u(t)}{t-\lambda}, \varphi(t)\right\rangle\left\langle\frac{\psi(x)}{x-\lambda}, v(x)\right\rangle-\frac{1}{D(\bar{\lambda})}\left\langle\frac{u(t)}{t-\bar{\lambda}}, \varphi(t)\right\rangle\left\langle\frac{\psi(x)}{x-\bar{\lambda}}, v(x)\right\rangle= \\
\frac{1}{2 D(\lambda)}\left\langle\frac{u(t)}{t-\lambda}, \varphi(t)\right\rangle\left\langle\frac{\psi(x)}{x-\lambda}, v(x)\right\rangle+\frac{1}{2 D(\bar{\lambda})}\left\langle\frac{u(t)}{t-\lambda}, \varphi(t)\right\rangle\left\langle\frac{\psi(x)}{x-\lambda}, v(x)\right\rangle- \\
\frac{1}{2 D(\bar{\lambda})}\left\langle\frac{u(t)}{t-\bar{\lambda}}, \varphi(t)\right\rangle\left\langle\frac{\psi(x)}{x-\bar{\lambda}}, v(x)\right\rangle-\frac{1}{2 D(\lambda)}\left\langle\frac{u(t)}{t-\bar{\lambda}}, \varphi(t)\right\rangle\left\langle\frac{\psi(x)}{x-\bar{\lambda}}, v(x)\right\rangle+ \\
\frac{1}{2}\left(\frac{1}{D(\lambda)}-\frac{1}{D(\bar{\lambda})}\right)\left\langle\frac{u(t)}{t-\bar{\lambda}}, \varphi(t)\right\rangle\left\langle\frac{\psi(x)}{x-\bar{\lambda}}, v(x)\right\rangle-
\end{gathered}
$$




$$
\frac{1}{2}\left(\frac{1}{D(\bar{\lambda})}-\frac{1}{D(\lambda)}\right)\left\langle\frac{u(t)}{t-\bar{\lambda}}, \varphi(t)\right\rangle\left\langle\frac{\psi(x)}{x-\bar{\lambda}}, v(x)\right\rangle .
$$

Denoting

$$
\begin{aligned}
I_{1}(\lambda) & \equiv\left\langle\frac{u(t)}{t-\lambda}, \varphi(t)\right\rangle \\
I_{2}(\lambda) & \equiv\left\langle\frac{\psi(x)}{x-\lambda}, v(x)\right\rangle,
\end{aligned}
$$

we then obtain

$$
\begin{aligned}
& \frac{1}{D(\lambda)}\left\langle\frac{u(t)}{t-\lambda}, \varphi(t)\right\rangle\left\langle\frac{\psi(x)}{x-\lambda}, v(x)\right\rangle- \frac{1}{D(\bar{\lambda})}\left\langle\frac{u(t)}{t-\bar{\lambda}}, \varphi(t)\right\rangle\left\langle\frac{\psi(x)}{x-\bar{\lambda}}, v(x)\right\rangle= \\
& \frac{1}{2}\left(\frac{1}{D(\lambda)}-\frac{1}{D(\bar{\lambda})}\right)\left[I_{1}(\lambda) I_{2}(\lambda)+I_{1}(\bar{\lambda}) I_{2}(\bar{\lambda})\right]+ \\
& \frac{1}{2}\left(\frac{1}{D(\lambda)}+\frac{1}{D(\bar{\lambda})}\right)\left[I_{1}(\lambda) I_{2}(\lambda)-I_{1}(\bar{\lambda}) I_{2}(\bar{\lambda})\right] \equiv \\
& \iint d x d t u(t) \overline{\varphi(t)} \psi(x) \overline{v(x)} K(x, t, k, \varepsilon) .
\end{aligned}
$$

Denoting

$$
\begin{aligned}
\mathcal{P}_{\varepsilon}(x) & =\frac{i \varepsilon}{x^{2}+\varepsilon^{2}} \\
\mathcal{H}_{\varepsilon}(x) & =\frac{x}{x^{2}+\varepsilon^{2}},
\end{aligned}
$$

then the kernel $K(x, t, k, \varepsilon)$ of the integral operator corresponding to the expression (3.4) takes the following form:

$$
\begin{aligned}
K(x, t, k, \varepsilon)=\left(\frac{1}{D(\lambda)}+\frac{1}{D(\bar{\lambda})}\right) \frac{1}{t-x}\left(\mathcal{P}_{\varepsilon}(x-k)-\mathcal{P}_{\varepsilon}(t-k)\right)- \\
\quad\left(\frac{1}{D(\lambda)}-\frac{1}{D(\bar{\lambda})}\right) \frac{1}{t-x}\left(\mathcal{H}_{\varepsilon}(x-k)-\mathcal{H}_{\varepsilon}(t-k)\right) .
\end{aligned}
$$

Taking into account the expression for $D(\lambda)$ and the assumption $\overline{\varphi(x)} \psi(x) \geq 0$, we see that

$$
\begin{aligned}
\left(\frac{1}{D(\lambda)}+\frac{1}{D(\bar{\lambda})}\right) & \rightarrow 2 \frac{1+\widetilde{\bar{\varphi}}(k)}{|D(k)|^{2}} \\
\left(\frac{1}{D(\lambda)}-\frac{1}{D(\bar{\lambda})}\right) & \rightarrow 2 i \pi \frac{\overline{\varphi(k)} \psi(k)}{|D(k)|^{2}}
\end{aligned}
$$


as $\varepsilon \rightarrow 0$, where $D(k) \equiv D(k+i 0)$ and the notation

$$
\tilde{f}(t) \equiv \lim _{\varepsilon \rightarrow 0} \int \mathcal{H}_{\varepsilon}(\tau-k) f(\tau) d \tau=\mathcal{\chi} \frac{f(\tau)}{\tau-k} d \tau
$$

is used for all $f$ such that the right-hand side makes sense.

The last limit exists almost everywhere on the real axis, see [6].

Consider

$$
\begin{aligned}
& \iint \frac{1}{t-x}\left(\mathcal{P}_{\varepsilon}(x-k)-\mathcal{P}_{\varepsilon}(t-k)\right) u(t) \overline{\varphi(t)} \psi(x) \overline{v(x)} d x d t \rightarrow \\
& \rightarrow i \pi \psi(k) \overline{v(k)} \widetilde{u \bar{\varphi}}(k)+i \pi u(k) \overline{\varphi(k)} \widetilde{\psi} \bar{v}(k)
\end{aligned}
$$

as $\varepsilon \rightarrow 0$ for almost all real $k$, see [6].

On the other hand,

$$
\begin{aligned}
& \iint \frac{1}{t-x}\left(\mathcal{H}_{\varepsilon}(x-k)-\mathcal{H}_{\varepsilon}(t-k)\right) u(t) \overline{\varphi(t)} \psi(x) \overline{v(x)} d x d t= \\
& \iint\left(\mathcal{H}_{\varepsilon}(x-k) \mathcal{H}_{\varepsilon}(t-k)+\mathcal{P}_{\varepsilon}(x-k) \mathcal{P}_{\varepsilon}(t-k)\right) u(t) \overline{\varphi(t)} \psi(x) \overline{v(x)} d x d t \rightarrow \\
& \rightarrow \widetilde{\psi \bar{v}}(k) \widetilde{u \bar{\varphi}}(k)+(i \pi)^{2} \psi(k) \overline{v(k)} u(k) \overline{\varphi(k)}
\end{aligned}
$$

as $\varepsilon \rightarrow 0$ for almost all real $k$, see [6].

Therefore, for almost all real $k$ the combination of (3.5) and (3.6) yields

$$
\begin{aligned}
\iint d x d t u(t) \overline{\varphi(t)} \psi(x) \overline{v(x)} K(x, t, k, \varepsilon) \rightarrow \\
\rightarrow 2 i \pi \frac{1+\widetilde{\bar{\varphi}}(k)}{|D(k)|^{2}}(\psi(k) \overline{v(k)} \widetilde{u \bar{\varphi}}(k)+u(k) \overline{\varphi(k)} \widetilde{\psi} \bar{v}(k))- \\
\quad-2 i \pi \frac{\overline{\varphi(k)} \psi(k)}{|D(k)|^{2}}\left(\widetilde{\psi \bar{v}}(k) \widetilde{u \bar{\varphi}}(k)+(i \pi)^{2} \psi(k) \overline{v(k)} u(k) \overline{\varphi(k)}\right) .
\end{aligned}
$$

Now let $u \in N_{i}^{0}$ and let $\operatorname{supp} u(x) \neq \mathbb{R}$. Consider the expression (3.7) on such functions $v$ that $\operatorname{supp} v=\mathbb{R} \backslash \operatorname{supp} u$. Since the term

$$
\left\langle\frac{u(x)}{x-\lambda}, v(x)\right\rangle-\left\langle\frac{u(x)}{x-\bar{\lambda}}, v(x)\right\rangle=2 \int d x \mathcal{P}_{\varepsilon}(x-k) u(x) \overline{v(x)} \rightarrow 2 i \pi u(k) \overline{v(k)}=0,
$$

we immediately obtain that in order for $u \in N_{i}^{0}$, it is necessary that $u \in H$ is a solution to the integral equation (3.1).

If, on the other hand, $u \in H$ is a solution to the equation (3.1), then by (3.7)

$$
\begin{aligned}
\frac{1}{2 i \pi}\left\langle\left[(L-\lambda)^{-1}-(L-\bar{\lambda})^{-1}\right] u, v\right\rangle \rightarrow & \\
& \rightarrow u(k) \overline{v(k)}+\frac{1+\widetilde{\bar{\varphi} \psi}(k)}{|D(k)|^{2}}(\psi(k) \overline{v(k) \widetilde{u \varphi}}(k)+u(k) \overline{\varphi(k)} \widetilde{\psi} \bar{v}(k))-
\end{aligned}
$$




$$
\begin{gathered}
\frac{\overline{\varphi(k)} \psi(k)}{|D(k)|^{2}}\left(\widetilde{\psi} \bar{v}(k) \widetilde{u \bar{\varphi}}(k)+(i \pi)^{2} \psi(k) \overline{v(k)} u(k) \overline{\varphi(k)}\right)= \\
u(k) \overline{v(k)}+\frac{1+\bar{\varphi} \psi(k)}{|D(k)|^{2}} \psi(k) \overline{v(k)} \widetilde{u \bar{\varphi}}(k)-\frac{\overline{\varphi(k)} \psi(k)}{|D(k)|^{2}}(i \pi)^{2} \psi(k) \overline{v(k)} u(k) \overline{\varphi(k)}=0,
\end{gathered}
$$

since the condition $\overline{\varphi(x)} \psi(x) \geq 0$ immediately implies that

$$
(1+\widetilde{\bar{\varphi} \psi}(k))^{2}+\pi^{2}(\overline{\varphi(k)} \psi(k))^{2}=|D(k)|^{2} .
$$

The next proposition provides a concise representation for the set of smooth vectors $\tilde{N}_{e}$ of the operator $L$. Note, that in order to prove this representation, we actually do not need to impose the restriction of $\overline{\varphi(x)} \psi(x) \geq 0$ for a.a. $x$.

Proposition 3.2. The set of smooth vectors $\tilde{N}_{e}$ of the operator $L$ admits the following representation:

$$
\begin{aligned}
\tilde{N}_{e}=\left\{u \in L_{2}(\mathbb{R})\right. & :\left\langle\frac{u(x)}{x-\lambda}, \psi(x)\right\rangle+ \\
& \left.\frac{1}{D(\lambda)}\left\langle\frac{u(t)}{t-\lambda}, \varphi(t)\right\rangle\left[a \pm i b-\left\langle\frac{\psi(x)}{x-\lambda}, \psi(x)\right\rangle\right] \in H_{2}^{ \pm}\right\},
\end{aligned}
$$

where

$$
a=-\frac{\operatorname{Re}\langle\varphi, \psi\rangle}{\|\varphi\|^{2}} ; \quad b=\frac{\left(\|\varphi\|^{2}\|\psi\|^{2}-(\operatorname{Re}\langle\varphi, \psi\rangle)^{2}\right)^{1 / 2}}{\|\varphi\|^{2}} .
$$

Proof. Denote

$$
\begin{aligned}
\tilde{\varphi} & =(a-i b) \varphi+\psi \\
\tilde{\psi} & =(a+i b) \varphi+\psi .
\end{aligned}
$$

Then $\langle\tilde{\varphi}, \tilde{\psi}\rangle=0$ and a straightforward algebraic check proves that

$$
\begin{gathered}
\mathcal{X}_{-}=\frac{1}{\|\tilde{\varphi}\|^{2}}\langle\cdot, \tilde{\varphi}\rangle \tilde{\varphi} \\
\mathcal{X}_{+}=\frac{1}{\|\tilde{\psi}\|^{2}}\langle\cdot \tilde{\psi}\rangle \tilde{\psi} \\
J=\mathcal{X}_{+}-\mathcal{X}_{-} ; \quad \alpha=\sqrt{\frac{1}{2 b}}\left(\|\tilde{\varphi}\| \mathcal{X}_{-}+\|\tilde{\psi}\| \mathcal{X}_{+}\right) .
\end{gathered}
$$

Combining these formulae with (3.2), we obtain: 


$$
\begin{aligned}
& \left\|\mathcal{X}_{-} \alpha(L-\lambda)^{-1} u\right\|=\sqrt{\frac{1}{2 b}}\|\tilde{\varphi}\|\left\|\mathcal{X}_{-}(L-\lambda)^{-1} u\right\|= \\
& \sqrt{\frac{1}{2 b}}\left|\left\langle\frac{u(x)}{x-\lambda}, \psi(x)\right\rangle+\frac{1}{D(\lambda)}\left\langle\frac{u(t)}{t-\lambda}, \varphi(t)\right\rangle\left[a-i b-\left\langle\frac{\psi(x)}{x-\lambda}, \psi(x)\right\rangle\right]\right| .
\end{aligned}
$$

An analogous computation yields the following result for $\mathcal{X}_{+} \alpha(L-\lambda)^{-1} u$ :

$$
\begin{aligned}
& \left\|\mathcal{X}_{+} \alpha(L-\lambda)^{-1} u\right\|=\sqrt{\frac{1}{2 b}}\|\tilde{\psi}\|\left\|\mathcal{X}_{+}(L-\lambda)^{-1} u\right\|= \\
& \sqrt{\frac{1}{2 b}}\left|\left\langle\frac{u(x)}{x-\lambda}, \psi(x)\right\rangle+\frac{1}{D(\lambda)}\left\langle\frac{u(t)}{t-\lambda}, \varphi(t)\right\rangle\left[a+i b-\left\langle\frac{\psi(x)}{x-\lambda}, \psi(x)\right\rangle\right]\right|,
\end{aligned}
$$

which completes the proof due to the definition of $\tilde{N}_{e}$, see (2.1).

The next theorem establishes the impact of the LRG condition (1.3) on the permitted spectral structure of the operator under investigation.

Theorem 3.3. Let $u$ be an eigenvector of the operator $L$ corresponding to the real eigenvalue $\lambda_{0}$. Let $\overline{\varphi(x)} \psi(x) \geq 0$ for a.a. $x \in \mathbb{R}$. Then the linear resolvent growth condition (1.3) implies that there exists an eigenvector $v$ of the operator $L^{*}$ corresponding to the same eigenvalue $\lambda_{0}$.

Proof. Without any loss of generality let $\lambda_{0}=0$. Then it is easy to see that $u$ is an eigenvector of $L$ corresponding to this eigenvalue if and only if $u(x)=\psi(x) / x \in$ $L_{2}(\mathbb{R})$ and the following condition holds:

$$
\int \frac{\overline{\varphi(x)} \psi(x)}{x} d x=-1 .
$$

We will prove that the LRG condition does not hold as long as $\varphi(x) / x \notin L_{2}(\mathbb{R})$ and is therefore not an eigenvector of the operator $L^{*}$. Consider

$$
|D(i \varepsilon)|^{2}=\left(1+\int \frac{\overline{\varphi(x)} \psi(x)}{x^{2}+\varepsilon^{2}} x d x\right)^{2}+\left(\int \frac{\overline{\varphi(x)} \psi(x)}{x^{2}+\varepsilon^{2}} \varepsilon d x\right)^{2} .
$$

Here

$$
\int \frac{\overline{\varphi(x)} \psi(x)}{x^{2}+\varepsilon^{2}} \varepsilon d x \rightarrow 0
$$

and

$$
\int \frac{\overline{\varphi(x)} \psi(x)}{x^{2}+\varepsilon^{2}} x d x \rightarrow \int \frac{\overline{\varphi(x)} \psi(x)}{x} d x
$$

as $\varepsilon \rightarrow 0$ by the Lebesque theorem; therefore, $D(i \varepsilon) \rightarrow 0$ as $\varepsilon \rightarrow 0$.

Since $\int \frac{\overline{\varphi(x)} \psi(x)}{x} d x=-1$, for (1.3) to be met it is therefore necessary (see (3.2)) 
that

$$
\limsup _{\varepsilon \rightarrow 0} \varepsilon^{2} \frac{\int \frac{|\varphi(x)|^{2}}{x^{2}+\varepsilon^{2}} d x \int \frac{|\psi(x)|^{2}}{x^{2}+\varepsilon^{2}} d x}{\left(\int \frac{\varepsilon^{2}}{x^{2}+\varepsilon^{2}} \frac{\psi(x)}{x} \overline{\varphi(x)} d x\right)^{2}+\left(\int \frac{\varepsilon}{x^{2}+\varepsilon^{2}} \psi(x) \overline{\varphi(x)} d x\right)^{2}} \leq C<\infty .
$$

By the Schwartz inequality,

$$
\left(\int \frac{\varepsilon^{2}}{x^{2}+\varepsilon^{2}} \frac{\psi(x)}{x} \overline{\varphi(x)} d x\right)^{2} \leq \int \frac{\varepsilon^{2}}{x^{2}+\varepsilon^{2}} \frac{|\psi(x)|^{2}}{x^{2}} d x \int \frac{\varepsilon^{2}}{x^{2}+\varepsilon^{2}}|\varphi(x)|^{2} d x
$$

where

$$
\int \frac{\varepsilon^{2}}{x^{2}+\varepsilon^{2}} \frac{|\psi(x)|^{2}}{x^{2}} d x \rightarrow 0
$$

as $\varepsilon \rightarrow 0$ by the Lebesque theorem. Taking into account that

$$
\int \frac{|\psi(x)|^{2}}{x^{2}+\varepsilon^{2}} d x \rightarrow \int \frac{|\psi(x)|^{2}}{x^{2}} d x
$$

as $\varepsilon \rightarrow 0$ by the same argument, we conclude that

$$
\left(\int \frac{\varepsilon^{2}}{x^{2}+\varepsilon^{2}} \frac{\psi(x)}{x} \overline{\varphi(x)} d x\right)^{2}=o\left(\varepsilon^{2} \int \frac{|\varphi(x)|^{2}}{x^{2}+\varepsilon^{2}} d x \int \frac{|\psi(x)|^{2}}{x^{2}+\varepsilon^{2}} d x\right)
$$

and therefore in our setting the condition (1.3) is satisfied only if

$$
\limsup _{\varepsilon \rightarrow 0} \varepsilon^{2} \frac{\int \frac{|\varphi(x)|^{2}}{x^{2}+\varepsilon^{2}} d x}{\left(\int \frac{\varepsilon}{x^{2}+\varepsilon^{2}} \psi(x) \overline{\varphi(x)} d x\right)^{2}} \leq C<\infty
$$

Let $h(x)$ be a bounded, continuous function of $x$ such that $h(x) \rightarrow 0$ as $x \rightarrow 0$, yet $\psi(x) / x h(x) \in L_{2}(\mathbb{R})$. Such choice of function $h$ is clearly always possible as long as $\psi(x) / x \in L_{2}(\mathbb{R})$. Then the denominator in (3.10) admits the following estimate:

$$
\begin{aligned}
& \left(\int \frac{\varepsilon}{x^{2}+\varepsilon^{2}} \psi(x) \overline{\varphi(x)} d x\right)^{2} \leq \\
& \int \frac{x^{2}}{x^{2}+\varepsilon^{2}} \frac{|\psi(x)|^{2}}{x^{2} h^{2}(x)} d x \int \frac{\varepsilon^{2}}{x^{2}+\varepsilon^{2}}|\varphi(x)|^{2} h^{2}(x) d x \leq C \int \frac{\varepsilon^{2}}{x^{2}+\varepsilon^{2}}|\varphi(x)|^{2} h^{2}(x) d x
\end{aligned}
$$

and it remains to show that as long as $\varphi(x) / x \notin L_{2}(\mathbb{R})$ the following holds:

$$
\int \frac{1}{x^{2}+\varepsilon^{2}}|\varphi(x)|^{2} h^{2}(x) d x=o\left(\int \frac{1}{x^{2}+\varepsilon^{2}}|\varphi(x)|^{2} d x\right)
$$

as $\varepsilon \rightarrow 0$, and hence the LRG condition (1.3) is violated. 
To observe this, choose $E \ll 1$ and fix $\delta_{0}=\delta_{0}(h, E)>0$ such that

$$
\frac{\int_{-\delta_{0}}^{\delta_{0}} \frac{1}{x^{2}+\varepsilon^{2}}|\varphi(x)|^{2} h^{2}(x) d x}{\int_{-\delta_{0}}^{\delta_{0}} \frac{1}{x^{2}+\varepsilon^{2}}|\varphi(x)|^{2} d x}<E .
$$

This is possible since $h(x) \rightarrow 0$ as $x \rightarrow 0$.

Now let us choose an $\varepsilon_{0}$ such that for all $\varepsilon<\varepsilon_{0}$

$$
\left(\int_{-\infty}^{-\delta_{0}}+\int_{\delta_{0}}^{\infty}\right) \frac{1}{x^{2}+\varepsilon^{2}}|\varphi(x)|^{2} d x \leq E \int_{-\delta_{0}}^{\delta_{0}} \frac{1}{x^{2}+\varepsilon^{2}}|\varphi(x)|^{2} d x,
$$

and, consequently,

$$
\left(\int_{-\infty}^{-\delta_{0}}+\int_{\delta_{0}}^{\infty}\right) \frac{1}{x^{2}+\varepsilon^{2}}|\varphi(x)|^{2} h^{2}(x) d x \leq E \int_{-\delta_{0}}^{\delta_{0}} \frac{1}{x^{2}+\varepsilon^{2}}|\varphi(x)|^{2} d x .
$$

Such a choice is clearly possible as long as $\varphi(x) / x \notin L_{2}(\mathbb{R})$.

Then for all $\varepsilon<\varepsilon_{0}$ one has the following estimate:

$$
\frac{\int \frac{|\varphi(x)|^{2} h^{2}(x)}{x^{2}+\varepsilon^{2}} d x}{\int \frac{|\varphi(x)|^{2}}{x^{2}+\varepsilon^{2}} d x} \leq C E(1+E) \ll 1,
$$

which completes the proof, since $E$ can be chosen to be arbitrarily small.

Remark 3.1. Under the assumptions of Theorem 3.3 the LRG property (1.3) clearly not only implies that $\lambda_{0}$ is an eigenvector of the adjoint operator $L^{*}$, but also that the corresponding eigenvectors are not orthogonal, i.e., $\langle u, v\rangle \neq 0$. Indeed, the estimate (3.10) implies in this case that $\langle\varphi / x, \psi / x\rangle \neq 0$, where $\varphi / x$ and $\psi / x$ are the eigenvectors of the operators $L^{*}$ and $L$, respectively, corresponding to the eigenvalue $\lambda_{0}=0$. The generalisation of (3.10) to the case when $\lambda_{0} \neq 0$ is trivial.

\section{The spectral structure in one special case of rich singular spectrum}

In this section we will study the operator $L$ defined by (1.1) in one special case. Namely, we impose a restriction that the singular subspace $N_{i}$ of $L$ and the singular subspace $N_{i}^{*}$ of $L^{*}$ are generated by the eigenvectors of the operators $L$ and $L^{*}$, respectively. We rigorously formulate this restriction in the form of a hypothesis.

Hypothesis $A$. Let $\overline{\varphi(x)} \psi(x) \geq 0$ for a.a. $x \in \mathbb{R}$. Let $\left\{u_{n}\right\}$ be a countable set of normalised eigenfunctions of the operator $L$ associated with a set of real eigenvalues $\left\{\lambda_{n}\right\}$ and $\left\{u_{n}^{*}\right\}$ be the corresponding set of normalised eigenvectors for the operator $L^{*}$. Let the singular subspaces $N_{i}=N_{i}^{0}$ and $N_{i}^{*}=N_{i}^{0}\left(L^{*}\right)$ coincide with the closed linear spans of $\left\{u_{n}\right\}$ and $\left\{u_{n}^{*}\right\}$, respectively. 
Remark 4.1. Note that the assumptions in the part of Hypothesis A concerning eigenvectors of the adjoint operator $L^{*}$ actually follow from Theorem 3.3. In particular, this theorem forces the operator $L^{*}$ to have the same pure point spectrum as the operator $L$ under the LRG condition (1.3). Therefore, an operator $L$ that satisfies the assumptions of Hypothesis A at the very least is not automatically disqualified from possessing the LRG property.

Now we are ready to prove the following series of results, describing the impact of the linear resolvent growth condition on the geometrical structure of the singular subspace and the angle between the singular and absolutely continuous subspaces.

Lemma 4.1. Let the functions $\varphi, \psi$ be such that Hypothesis A holds for the operator L. Then the LRG condition (1.3) implies the following:

$$
\left|\left\langle u_{n}, u_{n}^{*}\right\rangle\right| \geq c>0
$$

Proof. As in the proof of Theorem 3.3, we can show that if the operator $L$ has an eigenfunction $u_{n}$, corresponding to a real eigenvalue $\lambda_{n}$, then $u_{n}=c_{n} \frac{\psi(x)}{x-\lambda_{n}}$ and $D\left(\lambda_{n}\right) \equiv D\left(\lambda_{n}+i 0\right)=0$. Analogously, for the corresponding eigenfunctions $u_{n}^{*}$ of the operator $L^{*}$ we have $u_{n}^{*}=c_{n}^{*} \frac{\varphi(x)}{x-\lambda_{n}}$.

On the other hand, the LRG condition (1.3) implies that

$$
\frac{\varepsilon^{2} \int \frac{|\varphi(x)|^{2}}{\left(x-\lambda_{k}\right)^{2}+\varepsilon^{2}} d x \int \frac{|\psi(x)|^{2}}{\left(x-\lambda_{k}\right)^{2}+\varepsilon^{2}} d x}{\left(1+\int \frac{\overline{\varphi(x)} \psi(x)\left(x-\lambda_{k}\right)}{\left(x-\lambda_{k}\right)^{2}+\varepsilon^{2}} d x\right)^{2}+\left(\int \frac{\overline{\varphi(x)} \psi(x) \varepsilon}{\left(x-\lambda_{k}\right)^{2}+\varepsilon^{2}} d x\right)^{2}} \leq C<\infty
$$

uniformly with respect to $k$.

In absolutely the same way as in the proof of Theorem 3.3 we establish the following estimate:

$$
\begin{gathered}
\left(1+\int \frac{\overline{\varphi(x)} \psi(x)\left(x-\lambda_{k}\right)}{\left(x-\lambda_{k}\right)^{2}+\varepsilon^{2}} d x\right)^{2}=\left(\int \frac{\overline{\varphi(x)} \psi(x) \varepsilon^{2}}{\left(\left(x-\lambda_{k}\right)^{2}+\varepsilon^{2}\right)\left(x-\lambda_{k}\right)} d x\right)^{2} \leq \\
\int \frac{\varepsilon^{2}}{\left(x-\lambda_{k}\right)^{2}+\varepsilon^{2}} \frac{|\varphi(x)|^{2}}{\left(x-\lambda_{k}\right)^{2}} d x \int \frac{\varepsilon^{2}}{\left(x-\lambda_{k}\right)^{2}+\varepsilon^{2}}|\psi(x)|^{2} d x= \\
o\left(\int \frac{\varepsilon^{2}}{\left(x-\lambda_{k}\right)^{2}+\varepsilon^{2}}|\psi(x)|^{2} d x\right)
\end{gathered}
$$


as $\varepsilon \rightarrow 0$, and therefore the condition (4.2) is equivalent to the following one:

$$
\frac{\int \frac{|\varphi(x)|^{2}}{\left(x-\lambda_{k}\right)^{2}+\varepsilon^{2}} d x \int \frac{|\psi(x)|^{2}}{\left(x-\lambda_{k}\right)^{2}+\varepsilon^{2}} d x}{\left(\int \frac{\overline{\varphi(x)} \psi(x)}{\left(x-\lambda_{k}\right)^{2}+\varepsilon^{2}} d x\right)^{2}} \leq C<\infty .
$$

Taking into account that the left-hand side in (4.3) has a finite limit for each $k$ as $\varepsilon \rightarrow 0$, we arrive at the conclusion that the condition (4.3) is in turn equivalent to

$$
\frac{\left\|\frac{\varphi(x)}{x-\lambda_{k}}\right\|^{2}\left\|\frac{\psi(x)}{x-\lambda_{k}}\right\|^{2}}{\left|\left\langle\frac{\varphi(x)}{x-\lambda_{k}}, \frac{\psi(x)}{x-\lambda_{k}}\right\rangle\right|^{2}}=\frac{\int \frac{|\varphi(x)|^{2}}{\left(x-\lambda_{k}\right)^{2}} d x \int \frac{|\psi(x)|^{2}}{\left(x-\lambda_{k}\right)^{2}} d x}{\left(\int \frac{\overline{\varphi(x)} \psi(x)}{\left(x-\lambda_{k}\right)^{2}} d x\right)^{2}} \leq C<\infty,
$$

which completes the proof.

Lemma 4.2. Under the conditions of Hypothesis A, if the estimate (4.1) holds, then

(i) the set $\left\{u_{n}\right\}_{n=1}^{\infty}$ forms a Riesz basis in the singular subspace $N_{i}$;

(ii) the angle between the spectral subspaces $N_{e}$ and $N_{i}$ is positive.

Remark 4.2. Of course, the conditions of Lemma 4.2 also imply the dual result: the sequence $\left\{u_{n}^{*}\right\}_{n=1}^{\infty}$ forms a Riesz basis in the subspace $N_{i}^{*}$ and the angle between this subspace and $N_{e}^{*}$ is positive.

Proof. First, observe that under the conditions of Hypothesis A the following condition is satisfied:

$$
\left\langle u_{k}, u_{j}^{*}\right\rangle=0 \text { for all } j \neq k .
$$

Indeed, as was established in the course of the proof of Lemma 4.1,

$$
\begin{gathered}
u_{k}(x)=c_{k} \frac{\psi(x)}{x-\lambda_{k}} ; \quad u_{k}^{*}(x)=c_{k}^{*} \frac{\varphi(x)}{x-\lambda_{k}} \text { and } \\
\int \frac{\overline{\varphi(x)} \psi(x)}{x-\lambda_{k}}=-1 \text { for all } k .
\end{gathered}
$$

Therefore,

$$
\left(\lambda_{k}-\lambda_{j}\right) \int \frac{\overline{\varphi(x)} \psi(x)}{\left(x-\lambda_{k}\right)\left(x-\lambda_{j}\right)} d x=\int \frac{\overline{\varphi(x)} \psi(x)}{x-\lambda_{k}} d x-\int \frac{\overline{\varphi(x)} \psi(x)}{x-\lambda_{j}} d x=0
$$


Consider a vector $u \in N_{i}$ of the form

$$
u=\sum_{i=1}^{N} C_{i} u_{i}
$$

By Hypothesis A such vectors are dense in the subspace $N_{i}$. On the other hand, the operator $P_{n}$ defined by the identity

$$
P_{n} u \equiv \frac{\left\langle\cdot, u_{n}^{*}\right\rangle}{\left\langle u_{n}, u_{n}^{*}\right\rangle} u_{n}
$$

is well-defined and bounded on such vectors $u$. Hence, taking into account (4.4), its closure is the projection in $N_{i}$ onto the vector $u_{n}$ along the subspace $N_{i}^{(n)} \equiv$ $\bigvee_{i \neq n} u_{i}$

Since it is obvious that the condition (4.1) implies

$$
\left\|P_{n}\right\| \leq C
$$

uniformly with respect to $n$, the set $\left\{u_{n}\right\}_{n=1}^{\infty}$ forms a Riesz basis [2] in the subspace $N_{i}$.

In order to prove (ii), consider the operator $P$ on $H$ given by

$$
P=\sum_{k=1}^{\infty} \frac{\left\langle\cdot, u_{k}^{*}\right\rangle}{\left\langle u_{k}, u_{k}^{*}\right\rangle} u_{k}=\sum_{k=1}^{\infty} P_{k}
$$

The operator $P$ is well-defined on $H$. Indeed, $\left.P\right|_{N_{e}}=0$, since $N_{e}=H \ominus N_{i}^{*}$, and

$$
\sum_{i=1}^{\infty}\left|\left\langle P_{N_{i}^{*}} u, u_{i}^{*}\right\rangle\right|^{2} \leq C\left\|P_{N_{i}^{*}} u\right\|^{2}
$$

for all $u \in H$, since $\left\{u_{i}^{*}\right\}_{i=1}^{\infty}$ forms a Riesz basis in the subspace $N_{i}^{*}$ (see [2]).

Combined with (4.1) this clearly implies that the operator $P$ is bounded in $H$. On the other hand, since obviously $P u_{n}=u_{n}$ for all $n, P$ is the projection onto $N_{i}$ along $N_{e}$ and its boundedness implies that the angle between these two subspaces is positive.

Therefore, we arrive at the following result:

Theorem 4.3. Under the conditions of Hypothesis A, the linear resolvent growth condition (1.3) implies that the angles between the spectral subspaces $N_{e}$ and $N_{i}$; $N_{e}^{*}$ and $N_{i}^{*}$ are positive.

Remark 4.3. Consider a weaker 'limit' version of the LRG condition (1.3):

$$
\limsup _{\mid \operatorname{Im}}|\operatorname{Im} \lambda|\left\|(\mid L-\lambda)^{-1}\right\| \leq C
$$


It is easy to check that Theorem 3.3, Lemma 4.1 (and therefore Theorem 4.3) hold with the same proof if the LRG condition (1.3) is replaced by the weaker condition (4.7). Therefore, under Hypothesis A and (at least) condition (4.7), the effect of non-similarity to any self-adjoint operator may occur only due to an 'unfavourable' structure of the absolutely continuous part of the spectrum.

We now explicitly construct a family of operators $L$ of the class considered, i.e., a family of operators possessing the properties described in Hypothesis A.

Example 4.4. There exists a pair of functions $\varphi, \psi ; \overline{\varphi(x)} \psi(x) \geq 0$ a.e. on the real axis, such that for the corresponding non-self-adjoint operator $L$ Hypothesis $A$ holds.

Proof. Let $d_{k}=1 / 2^{k}, k=0,1,2, \ldots$ and consider the following definition:

$$
\psi_{k}(x) \equiv \varphi_{k}(x)= \begin{cases}1, & x \in\left[d_{k}+g_{k}, d_{k-1}-g_{k}\right] \\ 0, & \text { otherwise }\end{cases}
$$

where $g_{k} \ll d_{k}$. Let

$$
\varphi(x)=\sum_{k=1}^{\infty} a_{k} \varphi_{k}(x) ; \quad \psi(x)=\sum_{k=1}^{\infty} b_{k} \psi_{k}(x)
$$

where $a_{k}, b_{k}>0$ for all $k$. Then the condition $\overline{\varphi(x)} \psi(x) \geq 0$ is obviously satisfied everywhere on the real axis.

Furthermore, $\psi(x) / x \in L_{2}(\mathbb{R})$, which is clearly equivalent to the following: $\sum_{k=1}^{\infty} b_{k}^{2} / d_{k}<\infty$. The condition $\varphi \in L_{2}(\mathbb{R})$ is equivalent to $\sum_{k=1}^{\infty} a_{k}^{2} d_{k}<\infty$.

Consider the function $I(t)=\int \frac{\varphi(x) \psi(x)}{x-t} d x$ on the interval $t \in\left(d_{k}-g_{k+1}, d_{k}+g_{k}\right)$ for some fixed $k$. It is obviously well-defined on this interval and

$$
\begin{array}{r}
I(t)=\int_{d_{k} / 2+g_{k+1}}^{d_{k}-g_{k+1}} \frac{a_{k+1} b_{k+1}}{x-t} d x+\int_{d_{k}+g_{k}}^{2 d_{k}-g_{k}} \frac{a_{k} b_{k}}{x-t} d x+\sum_{i \neq k, i \neq k+1} \int_{d_{i}+g_{i}}^{2 d_{i}-g_{i}} \frac{a_{i} b_{i}}{x-t} d x \equiv \\
\equiv \int_{d_{k} / 2+g_{k+1}}^{d_{k}-g_{k+1}} \frac{a_{k+1} b_{k+1}}{x-t} d x+\int_{d_{k}+g_{k}}^{2 d_{k}-g_{k}} \frac{a_{k} b_{k}}{x-t} d x+\Sigma(t) .
\end{array}
$$

Now let $\sum_{k=1}^{\infty} a_{k} b_{k}<\infty$. Then a straightforward estimate shows that the function $\Sigma(t)$ is uniformly bounded on any of the intervals $\Delta_{k}=\left(d_{k}-g_{k+1}, d_{k}+g_{k}\right)$. Furthermore, it is easy to see that $\Sigma \in C^{\infty}\left(\Delta_{k}\right)$. Consider

$$
\Sigma(t)=\Sigma_{1}(t)+\Sigma_{2}(t) \equiv \sum_{i=1}^{k-1} \int_{d_{i}+g_{i}}^{2 d_{i}-g_{i}} \frac{a_{i} b_{i}}{x-t} d x+\sum_{i=k+2}^{\infty} \int_{d_{i}+g_{i}}^{2 d_{i}-g_{i}} \frac{a_{i} b_{i}}{x-t} d x
$$


In this decomposition, $\Sigma_{1}(t)$ is positive when $t \in \Delta_{k}$ and monotonically increasing; the function $\Sigma_{2}(t)$ is obviously negative and monotonically increasing as well; finally, $\sup _{t \in \Delta_{k}}\left|\Sigma_{2}(t)\right| \rightarrow 0$ as $k \rightarrow \infty$.

This argument clearly implies that there is one and only one solution to the equation

$$
I(x)=-1
$$

belonging to each of the intervals $\Delta_{k}$. Denote these points as $\left\{x_{k}\right\}_{k=1}^{\infty}$. By the definition of the functions $\varphi$ and $\psi$ it is clear then that $\varphi(x) /\left(x-x_{k}\right), \psi(x) /\left(x-x_{k}\right) \in$ $L_{2}(\mathbb{R})$ and are therefore eigenfunctions of the operators $L^{*}$ and $L$, respectively, corresponding to the real eigenvalues $\left\{x_{k}\right\}$. A straightforward application of Proposition 3.2 then proves that all the conditions of Hypothesis A are satisfied for the operators $L$ and $L^{*}$.

\section{ACKNOWLEDGEMENTS}

The author gratefully appreciates the financial support of IRCSET (Post-Doctoral Fellowships Programme) and RFBR (grant No. 03-01-00090).

The author is grateful to Dr D.J. Gilbert for fruitful discussions and to Professor S.N. Naboko and Dr M.M. Faddeev for their constant attention to his work.

\section{REFERENCES}

[1] C. Davis and C. Foiaş, Operators with bounded characteristic functions and their J-unitary dilatation, Acta Scientiarum Mathematicarum (Szeged) 32 (1-2) (1971), 127-39.

[2] I. Gohberg and M. Krein, An introduction to the theory of linear non-self-adjoint operators, Nauka, Moscow, 1965 (in Russian).

[3] A.V. Kiselev, On the similarity problem for the Friedrichs model operators, Vestnik St. Petersburg University series 1, 22 (4) (1998), 24-32 (in Russian).

[4] A.V. Kiselev and M.M. Faddeev, On the similarity problem for a class of non-self-adjoint operators, Vestnik St. Petersburg University series 1, 15 (3) (1996), 115-17 (in Russian).

[5] A.V. Kiselev and M.M. Faddeev, The similarity problem for non-self-adjoint operators with absolutely continuous spectrum, Functional Analysis and its Applications, 34, (2) (2000), 140-2.

[6] P. Koosis, Introduction to $H^{p}$ spaces, Cambridge University Press, 1980.

[7] S.N. Naboko, A functional model of the perturbation theory and its application to scattering theory, Proceedings of the Steklov Institute of Mathematics 147 (1980), 86-114.

[8] S.N. Naboko, On the singular spectrum of non-self-adjoint operator, Zapiski nauchnyh seminarov 113, (1981), 149-77 (English translation).

[9] S.N. Naboko, The conditions for similarity to unitary and self-adjoint operators, Functional Analysis and its Applications 18 (1984), 16-27.

[10] S.N. Naboko and M.M. Faddeev, Friedrichs model operators, similar to self-adjoint ones, Vestnik St. Petersburg University, series 4, 25 (1990), 78-82, (in Russian).

[11] N. Nikolski and S. Treil, Linear resolvent growth of rank one perturbation of a unitary operator does not imply its similarity to a normal operator, Journal d'Analyse Mathematique $\mathbf{8 7}$ (2002), 415-31.

[12] L.A. Sahnovich, Non-unitary operators with absolutely continuous spectrum, Izvestiya Akademii Nauk USSR (Math. USSR Izvestiya) 33 (1) (1969), 52-64.

[13] B. Sz.-Nagy and C. Foiaş, Analyse harmonique des operateurs de l'espace de Hilbert, Masson and Akad. Kiadó, Paris and Budapest, 1967.

[14] J. Van Casteren, Boundedness properties of resolvents and semigroups of operators, Acta Scientiarum Mathematicarum (Szeged) 48, (1-2) (1980), 189-94. 
[15] V.F. Veselov and S.N. Naboko, Characteristic function determinant and the singular spectrum of non-self-adjoint operator, Matematicheskii Sbornik 129 (171), (1) (1986), 20-39. 